\title{
Changing interpretations, stable genes: responsibilities of patients, professionals, and policy makers in the clinical interpretation of complex genetic information
}

Brian H. Shirts, $M D, P h D^{1}$, and Lisa S. Parker, $P h D^{2}$

\begin{abstract}
Except in rare mutation-inducing events, the primary sequence of an individual's somatic genome is static; however, the interpretations or risk predictions based on complex genetic tests now being introduced into the marketplace are rapidly changing. The reality of changing interpretations for stable test results creates questions for everyone involved in genetic testing including individuals, clinicians, laboratories, professional organizations, and regulators. Individuals should be aware that their relationship with laboratories providing genetic testing may be different from their relationship with their physician, especially in direct-to-consumer testing. Moreover, individuals may need to take the initiative to revisit their genetic test results periodically. Clinicians will need to learn how to read and interpret the results of complex genetic tests, remember that interpretations change over time, and understand when to refer patients to specialists and ask for second opinions and reinterpretation of genetic information. Testing laboratories should understand that they may be replacing the clinician as the direct contact for patients, and may have responsibility to inform clients of changes in test interpretation. At minimum, laboratories should make clear what their policies are regarding reinterpretation and allow tested individuals to seek outside interpretations of their genetic test results. Professional organizations and regulators have the responsibility to develop guidelines for clinicians, laboratories, and the general public. In the future, the interpretation of genetic tests may be relatively stable; until that time, the changing interpretation of static genetic test results will create an important set of professional and ethical challenges. Genet Med 2008:10(11):778-783.
\end{abstract}

Key Words: genetic policy, genetic testing, ethics of genetics, direct-to-consumer genetic testing, test result interpretation

\section{CHANGING INTERPRETATIONS, STABLE GENES}

Except in rare mutation-inducing events, the primary sequence of an individual's somatic genome is static. Thus, in contrast with clinical laboratory tests designed to monitor dynamic physiology, genetic tests produce unchanging results. However, the interpretations or risk predictions that come from complex genetic tests now being introduced to the marketplace are rapidly changing. The basic science of genetics on which genetic risk estimation is based has evolved rapidly and

From the ${ }^{I}$ Department of Pathology, University of Utah School of Medicine, Salt Lake City, Utah; and ${ }^{2}$ Center for Bioethics and Health Law, University of Pittsburgh, Pittsburgh, Pennsylvania.

Brain H. Shirts, MD, PhD, University of Utah Health Sciences Center, Department of Pathology, 15 North Medical Drive, Suite 1100, Salt Lake City, UT 84112. E-mail: brian.shirts@hsc.utah.edu.

Disclosure: The authors declare no conflicts of interest.

Submitted for publication July 17, 2008.

Accepted for publication August 25, 2008.

DOI: $10.1097 / G I M .0 b 013 e 31818 b b 38 f$ will create continually evolving applications to clinical and preventative genetics for the next generation and beyond.

Unlike simple or Mendelian traits, which usually involve one gene associated with a trait or illness, complex genetic traits usually involve interaction of multiple genes and environmental factors. Understanding genetic risk for complex genetic traits has been much more challenging than expected. Until recently, replication of new genetic association findings has been rare. ${ }^{1,2}$ Gregory Feero and colleagues at the National Human Genome Research Institute, summarized four limitations to preventative genetics:

1. Lack of information on how the prevalence and risk contribution of these markers vary across population groups;

2. Limited data on how the inheritance of multiple markers affects an individual's risk for various diseases;

3. Fragmentary information on how most genetic risk factors interact with environmental factors; and

4. Paucity of studies on common diseases that test the effect of interventions based on genetic risk factors. ${ }^{3}$ 
It is true that recent collaborative, multi-million dollar efforts have for the first time identified or confirmed reliable candidate genes for diseases such as diabetes and heart disease. ${ }^{4}$ Yet, risk alleles identified by these and other large genetic studies typically explain only a small portion of the heritable component of the disease, suggesting that there are many genes still to be discovered or genetic and environmental interaction factors to be invoked before truly accurate risk predictions can be made for these conditions.

Despite these limitations, commercial laboratories are starting to offer genetic tests to clinicians and directly to the public that use the available information to calculate genetic risk for complex traits. Previous discussion about issues specific to whole genome testing have focused on several issues associated with their marketing as direct-to-consumer tests "for informational purposes only," presumably to avoid their being exposed to the scientific and technical rigors of medical tests. 5,6 Companies are addressing these issues, and it is becoming clear that complex genetic testing-both directly marketed tests and testing ordered by clinicians-will continue to increase. There is little discussion about the practical and ethical issues regarding how patients, clinicians, and regulatory organizations should deal with the changing interpretation of static test results from complex genetic tests.

\section{GENETIC TESTS AS AN EXCEPTION OR AS THE EXTREME IN A SPECTRUM?}

The interpretation and utility of all laboratory test results, genetic and traditional, change over time. Comparing genetic tests with other laboratory tests reveals how they may be unique. Compare a genetic panel for a complex disease to two different classes of common medical tests: basic clinical chemistry tests, such as serum creatinine, and simple imaging studies, such as a CT scan. The results of nongenetic tests are known to represent the clinical picture at a single point in time. Clinical chemistries are usually considered valid for hours to days. CT scan images may be considered valid for months if the correct images are taken. The interpretations of those results are generally considered valid and up-to-date within the given time frame and not questioned. This is because medical understanding of the tests themselves changes gradually over decades. A serum creatinine level or a CT scan might be interpreted differently today than it was a decade ago, but not differently than it was a few days or months ago. In other words, the interpretation of a test result has a much longer valid life than the test result (laboratory values or images) itself. Genetic tests reverse this framework. Once a genetic test on somatic tissue is done, the results are valid for the lifetime of the individual, and perhaps longer as genetic data may have relevance for the individual's offspring and other relatives. However, given the current pace of genetic research, the meaning of genetic tests for complex conditions could very well change over the course of an individual's lifetime. This is true to varying degrees for all genetic tests whether they evaluate one variant, a panel of polymorphisms, or the whole genome.
Genetic tests of single genes are likely to be done to investigate a simple condition which has likely been extensively studied and for which a relatively stable body of knowledge is available; the interpretation of this test might change over decades. On the other hand, information about complex genetic risk as assessed by genetic panels and whole genome scans is constantly changing; the interpretation might change substantially over months and is likely to be very different in 2 to 5 years.

For all nontumor genetic tests, the actual genotype or sequence result will stay stable. Once someone has a single gene sequenced, he should never need that gene sequenced again; likewise, once someone has a comprehensive whole genome scan (currently, commercial whole genome scans may not be considered comprehensive as they do not necessarily cover variants that may be found to be important in the future, but for practical purposes may be comprehensive enough and will certainly be comprehensive in the near future), she should never need to give a DNA sample for another genetic test in her lifetime. The reality of changing interpretations creates questions for everyone involved in genetic testing. How should individuals, medical professionals, testing laboratories, professional organizations, and societal regulatory bodies deal with changing interpretations of stable test results?

\section{INDIVIDUALS AND PATIENTS}

Some ethical issues in the interpretation of genetic data arise from changes in the relationships between the genetic testing laboratory and the person being tested, and between the laboratory and the person ordering the tests. In many clinical settings, the laboratory is primarily responsible for making sure the result is accurate, and the clinician is primarily responsible for the interpretation. Thus, the laboratory has a primary responsibility to the clinician ordering the test, and the clinician has a primary responsibility to the patient. In an era of personalized medicine, it is likely that the laboratory will have a more direct relationship with individuals. Direct-to-consumer testing is emerging as the norm for large scale genetic testing for disease risk information. This changes the usual hierarchy of laboratory to clinician to patient responsibility and makes laboratories directly responsible to individuals.

Many ethical issues with direct-to-consumer testing have been noted by others. ${ }^{6,7}$ Direct-to-consumer marketing of complex genetic tests became a sufficiently prominent and pressing issue that the Federal Trade Commission issued a statement in 2006 providing information about the nature of genetic tests and warning about potential false claims. ${ }^{8}$ The American College of Medical Genetics (ACMG) most recent recommendations on direct-to-consumer testing include five minimum criteria: (1) a professional should be involved in ordering and interpreting the tests; (2) the consumer is fully informed about the test's capabilities and limitations; (3) the scientific evidence is clearly stated; (4) the laboratory should be accredited by CLIA and state agencies; and (5) privacy concerns must be addressed. ${ }^{9}$ At some direct-to-consumer genetic test producers, the promise of the tests are either oversold by 
companies or viewed with insufficient understanding and skepticism on the part of consumers, whereas other direct-toconsumer testers are honestly trying to make cutting edge genetic information available to the public and are committed to the highest quality science and ethical dissemination of genetic information.

Nevertheless, individuals should be aware that their relationship with testing companies is different from their relationship with their physicians. Where a personal physician who knows about a change in the interpretation of personal health data would clearly be expected to follow-up with a patient, a company may have no similar responsibility to follow-up with updated information if it is not spelled out in a company's promises or contracts. Individuals should exercise caution to discern what laboratory companies are providing them, as well as the time frame of the company's commitment to updating genetic test result interpretation. Individuals should also be cautious about locked-in business models where, once the testing is done, individuals subscribe to an interpretation service from the same company and are charged periodically to get updated risk estimates without having access to sufficient genetic data to enable them to easily seek outside analysis.

Permitting individuals to seek independent interpretations by their personal physicians, however, may not be a panacea. As the FTC and CDC note "because of the complexities involved in both the testing and the interpretation of the results, genetic tests should be performed in a specialized laboratory, and the results should be interpreted by a doctor or trained counselor who understands the value of genetic testing for a particular situation." ${ }^{8}$ Individuals should be aware, however, that their personal physicians may not be qualified to update test interpretations and may not even be able to act as the individual's health agent to access the raw test results or laboratory representatives if there are questions about current validity, changing interpretation, and clinical relevance. Even when results are interpreted by a doctor or genetic counselor trained to interpret complex genetic tests, individuals should be aware that they should periodically revisit the results and ask that their risk be recalculated in light of newer findings.

\section{MEDICAL PROFESSIONALS AND CLINICIANS}

In the practice of taking a family history and medical history, clinicians have a well-established tradition of obtaining information that has long-term health consequences and integrating it into clinical decisions. Genetic risk can be directly related to familial risk and similar to other past experiences in risk modeling. However, family history and medical history have usually been used by clinicians as blunt tools. The integration of genetic information provides an opportunity to refine risk assessment and related clinical decision-making tools. However, while the meaning of genetic test results remains in transition, the possibility is great that patients and even clinicians are overconfident of the precision and value of genetic testing as a tool.
Because it is so new, most medical schools have not begun to teach how a clinician could potentially incorporate genetic information into decision-making for common diseases or interpret risk data from a whole genome scan or complex risk panel. ${ }^{10}$ Clinicians should become more comfortable with genetic tests and begin to educate themselves about what clinically useful information their patients may reasonably expect from laboratory tests and what the tests' limitations are. Clinicians will need to know when a genetic screening test interpretation can be considered sound for clinical decision-making and when additional genetic or nongenetic testing is necessary.

When current health care decisions are made on the basis of past test results, it is a matter of clinician responsibility to ensure that the interpretation of that test result still holds. However, it has not been established how far the clinician's responsibility extends when it comes to additional interpretation and communication of genetic test results that preexisted the specific clinician-patient relationship and that will last after this relationship has ended. ${ }^{11-13}$ Court cases involving physician responsibility to the children of their patients may provide some guidance about legal liability. ${ }^{14,15}$ Legal liability will eventually be decided by regulators and the courts; however, anticipation of potential issues emphasizes the need for clinician aids in genetic interpretation. Given the clinician's responsibility to ensure the interpretation of test results is current for clinical decisions, it is very important that clinicians be afforded adequate support systems necessary, such as referrals to genetics specialists, access to necessary laboratory information, and electronic databases of guidelines possibly with decision support tools that can be linked to patient test results.

For common tests, such as the serum chemistries or imaging studies mentioned before, an expert may be called in to give a second opinion about interpretation of the test if there is any uncertainty. In the context of comprehensive whole genome scanning, there should be no need to repeat the same tests, but recalculation of risk using new algorithms may be critical for the accurate up-to-date interpretation of tests. Clinicians should not be afraid to ask for a second opinion, consult genetics specialists, or contact genetic testing laboratories directly if they or their patients have difficulty in understanding or accessing their genetic information.

Clinicians rely on accurate laboratory medicine reports to appropriately inform their patients and find additional information about new tests, especially when the clinicians themselves are not familiar with a new test. Guidelines regarding appropriate documentation to accompany results from new tests should be developed so that the documentation can be easily understood by clinicians. (Suggested content for such documentation is discussed below.) If multiple variants are being tested for several traits at once, such as in whole genome scans, the amount of information will be large enough that new techniques of reporting information to both individuals and clinicians will need to be developed and standardized.

In addition to test reports from testing laboratories, webbased databases designed for clinicians that specifically address 
common genetic variation are being developed. These aim to aid clinicians in search of quality information about genetic risk and disease. There is also a move to develop clinical decision support tools that are linked to electronic medical records. These systems offer advantages of being easily updated and may be necessary for both primary care providers and genetic specialists to navigate the vast quantities of information that genetic testing will potentially provide. Further development of these resources may be necessary because as the accumulation of genetic information accelerates even geneticists may be unable to keep abreast of changing interpretations in areas not directly related to their specialty.

\section{LABORATORIES AND TESTING COMPANIES}

Following the logic of lab to clinician and clinician to patient responsibility, older guidelines from the American College of Medical Genetics stressed the impracticality of laboratories recontacting patients to inform them of changes in genetic information and placed the responsibility on clinicians to keep abreast of changes in genetic information. ${ }^{12}$ This was not seen as an undue burden because the genetic tests available investigated one or two genes and tested specific simple traits. However, the ACMG recently published new guidelines modifying this position and suggested that for rare variants testing laboratories may be in the best position to modify previous interpretations. ${ }^{16}$ In its statement, the ACMG recognized the possibility that knowledge of changes in interpretation may be difficult to access and communicate for anyone other than specialists. Although this change of guidelines specifically addressed rare genetic tests, the rationale for the change was the restricted nature of the knowledge and the inability of all clinicians to have easy access to the relevant data. The acknowledgment that not all clinicians could or should be expected to be up-to-date on all changes in genetic understanding is equally applicable to genetic risk calculations for complex disease. Certainly a primary care physician should have the general skills necessary to interpret and use the results of genetic tests, but laboratories or companies that perform complex genetic tests will be in a much better position to know when a test's interpretation is out-of-date and reinterpretation necessary. Furthermore, if there is no clinician intermediary between the laboratory and the individual, the testing laboratory logically should assume the responsibility of negotiating with those tested a method to inform them of changes in interpretation.

The issue of recontacting individuals about changing interpretations of genetic tests is the subject of ongoing debate. ${ }^{11,13,17}$ This debate will undoubtedly continue and become more complicated when large numbers of genetic variants are tested and in situations where the physician intermediary is removed from the relationship between laboratory and individual.

To obviate concerns about recontacting, laboratories performing tests for complex genetic traits can preemptively address this issue by defining appropriate expectations in their advertising information and appropriately acknowledging limitations in test descriptions. Laboratories should make it clear to individuals desiring such testing that the interpretation of their results is likely to change. In a testing situation, such as whole genome scans, where an ongoing subscription will be necessary for updated risk calculations, this fact should be clear from the beginning, and the long-term costs associated with such a subscription should be disclosed. Laboratories should also create reports with qualifiers and warnings indicating that interpretations, including risk predictions, are only valid at the time of the report and recommending how and when the interpretations should be updated. Even with these disclosures, situations may arise in which a change in interpretation is so dramatic that there is an ethical obligation for a laboratory to attempt to contact and inform individuals tested regardless of previous disclaimers or lapsed subscription status.

Directors of clinical laboratories are obligated by CLIA and professional standards to provide documentation including pertinent information required for interpretation with reported test results. ${ }^{16,18}$ For more complicated or rare tests, the documentation is understandably more comprehensive. The ACMG guidelines for genetic sequence variations recommends that reports of genetic tests include information listing the specific sequences tested; information indicating the level of existing knowledge about variants found; discussion of the scientific basis of interpretations including citations to literature; indication of methodology with sensitivity, reliability, and limitations; and information about gene expression that may influence disease treatment and prognosis. ${ }^{16}$

Because the genetic variants analyzed by whole genome scans are static, providing standardized information that can be interpreted and confirmed at a later date by a third party is vitally important. For whole genome scans, there is no current standard for formatting the raw genetic data or for documentation of risk assessment models. Current guidelines for genetic testing emphasize the importance of utilizing standardized terminology from established databases. ${ }^{16}$ Standards developed by scientific journals for reporting variants so that they can be understood by a third party should suffice until improved standards are developed in light of experience. Appropriate standards might include reporting NCBI dbSNP build, rs number, and strand direction for each variant or location of variants within reference sequence from the NCBI refseq database. Ideally, such data should be easily incorporated into third party electronic clinical decision support tools. Even though the individuals being tested may not be able to interpret the results themselves, the genetic information is theirs, and they should be able to take the information to obtain a second opinion, as they might do with any other test. It appears at this time that the business model of some companies offering whole genome scans is a locked-in service model. ${ }^{19}$ This business model, though it may be lucrative, is not consistent with ethical reporting of lab results unless all uninterpreted genetic information is made available for second opinions. Instead of seeking profit by utilizing this locked-in model, 
companies should strive to retain clients by providing the best service with transparent risk calculations and understandable reports so that it is clear both what interpretation has been done and how long the information is valid.

\section{PROFESSIONAL ORGANIZATIONS}

Professional organizations have the responsibility to develop guidelines about genetic testing in specific conditions relevant to their members, and to communicate those guidelines to practitioners in their fields. Once there are established professional guidelines for using genetic data in risk calculations, such as those already in place for risk of breast, ovarian, and other cancers, ${ }^{20,21}$ there is no place for proprietary risk formulas, which avoid legitimate scientific debate about the validity of the risk assessment. However, when there are no such professional guidelines, it is hard to make a strong argument against the utilization of proprietary risk formulations.

With regard to changing interpretations, guidelines should be revisited periodically to address several issues: (1) When is the level of evidence sufficient to analyze genetic data for specific clinical conditions, and when is the evidence so inconclusive that analysis of genetic data should wait? (2) What level of preexisting familial and environmental risk would make genetic analysis recommended, and when would it be optional? (3) What are the best formulas for calculating risk? (4) When have sufficient data been produced to change previously recommend risk calculations? (5) When there is a change in risk calculation, what are the criteria for determining whether previously tested individuals should be contacted to inform them of a modified risk, and when should it be left up to individuals to initiate such follow up? Once guidelines are created, professional organizations should actively disseminate their guidelines and try to ensure that they are appropriately incorporated into electronic clinical decision support tools.

\section{REGULATORY BODIES}

Regulators in government and institutional roles should be aware of the issues involved in genetic testing and provide general guidelines for laboratories, clinicians, and individuals. Currently genetic tests, including whole genome genetic tests, are available to individuals both directly from laboratory companies and through clinicians. The decision as to which tests are direct-marketed and which tests are ordered through clinicians is now decided mainly by the companies offering the tests and by consumers. As testing becomes more common, it may be clear that some commercial laboratories are consistently using inaccurate or outdated information, presenting misleading or manipulative advertizing or reports, or not cooperating appropriately with clients and clinicians to reinterpret test results when necessary. Any of these situations could necessitate tighter regulation of genetic testing, which could include requiring that a clinician order certain genetic tests or formaliz- ing the responsibilities of genetic testing laboratories regarding documentation and reinterpretation.

The recent debate surrounding New York and California issuing "cease and desist" letters to certain genetic testing companies highlights the fact that it is not clear where and how to regulate the industry. These states have questioned the scientific validity of the interpretations being offered, which is emphasized by rapid changes in the understanding of genetic risk. Testing companies argue that their tests are based on the best current scientific understanding, and that consumers should not have to wait decades for the final scientific conclusion. If standards for reinterpretation of genetic tests are established, these may alleviate some of the concern about absolute scientific validity since interpretations will be given with clear explanation of their transitory nature.

\section{CONCLUSION}

The growing body of scientific evidence will be useful for clinical applications and public health to the degree that individuals, clinicians, laboratories, professional organizations, and regulatory bodies act in scientifically and ethically sound ways with regard to genetic data. Because genetic information generated by a test is more static than its interpretation, instead of redoing tests and producing new test results with new interpretations, the interpretation of original test results will need to be periodically revisited. At some point, it may be the case that the interpretation of genetic tests changes minimally through the course of a person's lifetime, but that will not happen in the near future. Until that time, the changing interpretation of static genetic test results will create a unique set of professional and ethical problems to be addressed by all parties involved.

\section{ACKNOWLEDGMENTS}

Supported by Grants from MH074265 (to BHS) and ACISRMH071944 (to LSP).

\section{References}

1. Gorroochurn P, Hodge SE, Heiman GA, Durner M, Greenberg DA. Non-replication of association studies: "pseudo-failures" to replicate? Genet Med 2007;9:325-331.

2. Khoury MJ, Little J, Gwinn M, Ioannidis JP. On the synthesis and interpretation of consistent but weak gene-disease associations in the era of genome-wide association studies. Int J Epidemiol 2007;36:439-445.

3. Feero WG, Guttmacher AE, Collins FS. The genome gets personal-almost. JAMA 2008;299:1351-1352.

4. Consortium WTCC. Genome-wide association study of 14,000 cases of seven common diseases and 3,000 shared controls. Nature 2007;447:661-678.

5. Offit K. Genomic profiles for disease risk: predictive or premature? JAMA 2008;299: 1353-1355.

6. Wasson K, Cook ED, Helzlsouer K. Direct-to-consumer online genetic testing and the four principles: an analysis of the ethical issues. Ethics Med 2006;22:83-91.

7. Wade $\mathrm{CH}$, Wilfond BS. Ethical and clinical practice considerations for genetic counselors related to direct-to-consumer marketing of genetic tests. Am J Med Genet C Semin Med Genet 2006;142C:284-292; discussion 293.

8. Commission FT. At-home genetic tests: a healthy dose of skepticism may be the best 
prescription. Available at: http://www.ftc.gov/bcp/edu/pubs/consumer/health/hea02. shtm. Accessed July 15, 2008.

9. Genetics Acom. Acmg statement on direct-to-consumer genetic testing. Available at: http://www.acmg.net/staticcontent/staticpages/dtc_statement.pdf. Accessed July 15, 2008.

10. Telner DE, Carroll JC, Talbot Y. Genetics education in medical school: a qualitative study exploring educational experiences and needs. Med Teach 2008;30:192-198.

11. Letendre M, Godard B. Expanding the physician's duty of care: a duty to recontact? Med Law 2004;23:531-539.

12. Hirschhorn K, Fleisher LD, Godmilow L, et al. Duty to re-contact. Genet Med 1999; $1: 171-172$.

13. Fitzpatrick JL, Hahn C, Costa T, Huggins MJ. The duty to recontact: attitudes of genetics service providers. Am J Hum Genet 1999;64:852-860.

14. Pate VT. Wests South Rep 1995;661:278-282.

15. Safer V. Estate of pack. Atl Rep 1996;677:1188-1193.

16. Richards CS, Bale S, Bellissimo DB, et al. ACMG recommendations for standards for interpretation and reporting of sequence variations: revisions 2007. Genet Med 2008; 10:294-300.

17. Hunter AG, Sharpe N, Mullen M, Meschino WS. Ethical, legal, and practical concerns about recontacting patients to inform them of new information: the case in medical genetics. Am J Med Genet 2001;103:265-276.

18. Center for medicaid and medicare services dohahs. $42 \mathrm{cfr} 493.1407$ standard; laboratory director responsibilities. 2007. Available at: http://edocket.access.gpo.gov/ cfr_2004/octqtr/pdf/42cfr493.1407.pdf. Accessed July 15, 2008.

19. Hamilton D. Will 23andme and navigenics lock up your genome and charge you for the key? Venturebeat 2007.

20. Lancaster JM, Powell CB, Kauff ND, et al. Society of Gynecologic Oncologists Education Committee statement on risk assessment for inherited gynecologic cancer predispositions. Gynecol Oncol 2007;107:159-162.

21. Smith RA, Cokkinides V, Brawley OW. Cancer screening in the United States, 2008: a review of current American Cancer Society guidelines and cancer screening issues. CA Cancer J Clin 2008;58:161-179. 